\title{
Radio Proximity Fuze Design
}

\author{
By Wilbur S. Hinman, Jr., and Cledo Brunetti
}

\begin{abstract}
The general principles governing the design of radio proximity fuzes are presented. The paper deals primarily with fuzes for smooth-bore projectiles, ${ }^{*}$ such as bombs, rockets and mortars. Illustrations and descriptions of the various fuzes in this eategory which were developed during World War II are given. Within security regulations, there is a reasonably detailed discussion of the performance and construction of fuze components, such as the oscillator, the amplifier, the antennas, the power supply and the safety and arming mechanisms. There is also a brief description of production practices and problems and methods of inspection and quality control.
\end{abstract}

\section{Introduction}

Electronic arts are generally linked to intelligence, and the radio proximity fuze follows the pattern. Intelligence might be described as the ability to adopt or change a course of action according to the circumstances of the moment to give the most effective result without external influence. This sense, which is the prime feature of the radio proximity fuze, fulfills one of the dreams of the ordnance man and provides a fuze that increases weapon effectiveness many fold. If a projectile can be made to explode at its closest approach to an airplane target, the effective size of the target is increased greatly; if a projectile can be made to explode above the ground, fragments are sprayed out over a wide area instead of being buried in the ground at the point of contact or directed harmlessly upward.

The radio proximity fuze was developed to meet these requirements. It is an extremely small transmitting station with a receiver to detect any reflections of the transmitted wave.

\footnotetext{
*Distinction is made between the application to rotating and nonrotating projectiles. The difference lies principally in factors such as power supply, arming mechanism, and physical structure. The first two considerations are dependent primarily on the conditions of acceleration and motion. The physical structure is governed by the size and contour of the projectile.
}

It works on the Doeppler principle, and operates whenever the amplitude of the reflected signals exceed a predetermined value. There is the additional restriction that the velocity of approach must be such that the resulting Doeppler frequency lies in the relatively narrow frequency band for which the fuze was designed.

As certain specific details of fuze construction still fall under the heading of classified information, it is necessary to introduce some generalities in this paper in order to meet security regulations.

\section{Contents}

I. Introduction $\ldots \ldots$

II. General description . . . . . . . . . . . 3

III. Oscillator . . . . . . . . . . . . . . . . . 4

IV. Amplifier . . . . . . . . . . . . . . 4

V. Radiation, reflection, and operating range . . 7

VI. Power supply . . . . . . . . . . . . . . 8

VII. Safety and arming . . . . . . . . . 10

VIII. Tubes and other components . . . . . . . 11

IX. Production and laboratory testing . . . . . . 11

X. Field testing . . . . . . . . . . . 12 
The impact of this new application of electronics on military science and tactics is enormous. It is the counter-weapon to airplanes and robots raiding ships or cities. It is a new offensive weapon for airplanes. When used against entrenched troops or troops on the march, it provides a new order of importance for the shell, bomb, or rocket with which it is used. Some of the foremost military leaders have said that it precluded troop movement in the open. Remembering that most military tactics depend upon the movement of troops and supplies for sudden attacks and quick support of defenses, one can begin to see the importance of the application.

The work on proximity fuzes started in this country in August 1940 under Office of Scientific Research and Development auspices. In the spring of 1941, in order to press the development with maximum efficiency, the Navy assumed responsibility for the development and procurement of proximity fuzes for rotating projectiles, such as antiaircraft and artillery shells, while the Army directed the development of fuzes for nonrotating projectiles, such as bombs, rockets, and mortars. This paper deals with the Army phase of proximity fuze development. Some of these fuzes mounted on projectiles are shown in figure 1.

The existence of an effective radio fuze was one of the best-kept secrets of the war. Scientists of all countries had the basic concept, but only the United States and her Allies were able to work from the concept to practical and effective operation and production, for the radio proximity fuze is a paradox. Simple in general theory, it is extraordinarily complex in the variety of applied arts that are combined in its practical aspects.

Much of the necessary engineering and development work was devoted to meeting exacting military requirements, such as (1) the fuze must fit existing projectiles using the same fuze well as the mechanical fuzes, and it must not project more than say 5 inches beyond the well; (2) it must be capable of withstanding long storage conditions at high and low temperatures and of operating under these conditions; (3) it must not alter the ballistic characteristics of the projectile; and (4) it must meet other strict requirements relating to time of activation, safety, and performance.

The size limitations for radio proximity fuzes were fixed by military necessity. One might think that in the case of a 500- or 1,000-pound bomb, a few extra cubic inches or an extra pound or two would not be important, but bombs and bomb bays were already designed and in use. A fuze projecting so far as to prohibit loading a fuzed bomb into the bomb bay would be useless. Ballistic tables for all bombs were available, and if the radio fuze affected the trajectory and changed the bombing tables appreciably, use of the fuze would be prejudiced. For the smaller projectiles, such as the trench mortar, size and weight limitations were even more severe. One of the 81-mm mortar shells weighs approximately 8 pounds, and unless the radio fuze is light and fairly well streamlined, the range of the mortar is reduced-a severe handicap to its military effectiveness.

Other basic difficulties are readily apparent. Mortar fuzes must withstand acceleration of $10,000 \mathrm{~g}$. In the fuzes for bombs and rockets, the projectile vibrations caused by flight velocities approaching or exceeding the speed of sound required great rigidity in the design of the fuzes and their components. Some idea of the energy producing these vibrations may be had by considering conditions at terminal velocity. Air friction and turbulence prevent further acceleration and do work on the bomb, which work appears as vibration and heat. For a 1,000-pound bomb at a terminal velocity of $1,000 \mathrm{ft} / \mathrm{sec}$, the rate of energy dissipation is $1,000,000 \mathrm{ft}-\mathrm{lb} / \mathrm{sec}$, or 1,356 kilowatts. Taking into account the desirable requirements of low cost and small size, and with the further realization that an extraordinary degree of electronic stability is needed for satisfactory operation, it is evident that the performance requirements for these electronic devices are very strict.

These restrictions required the development of new, very small and strong components. It is fortunate that "small" and "strong" go together. Weight is proportional to the cube of the linear dimension, while the supporting area is proportional to the square of the linear dimension. Thus, dimensional reduction by a factor of 10 results in an object whose strength under acceleration is increased by 10. Resistance against centrifugal forces and bending moments is also greatly increased by a reduction in size. This is the reason that electronic components, generally considered delicate, can be constructed so as to withstand the high vibratory and accelerating forces which are developed in the various projectiles. 


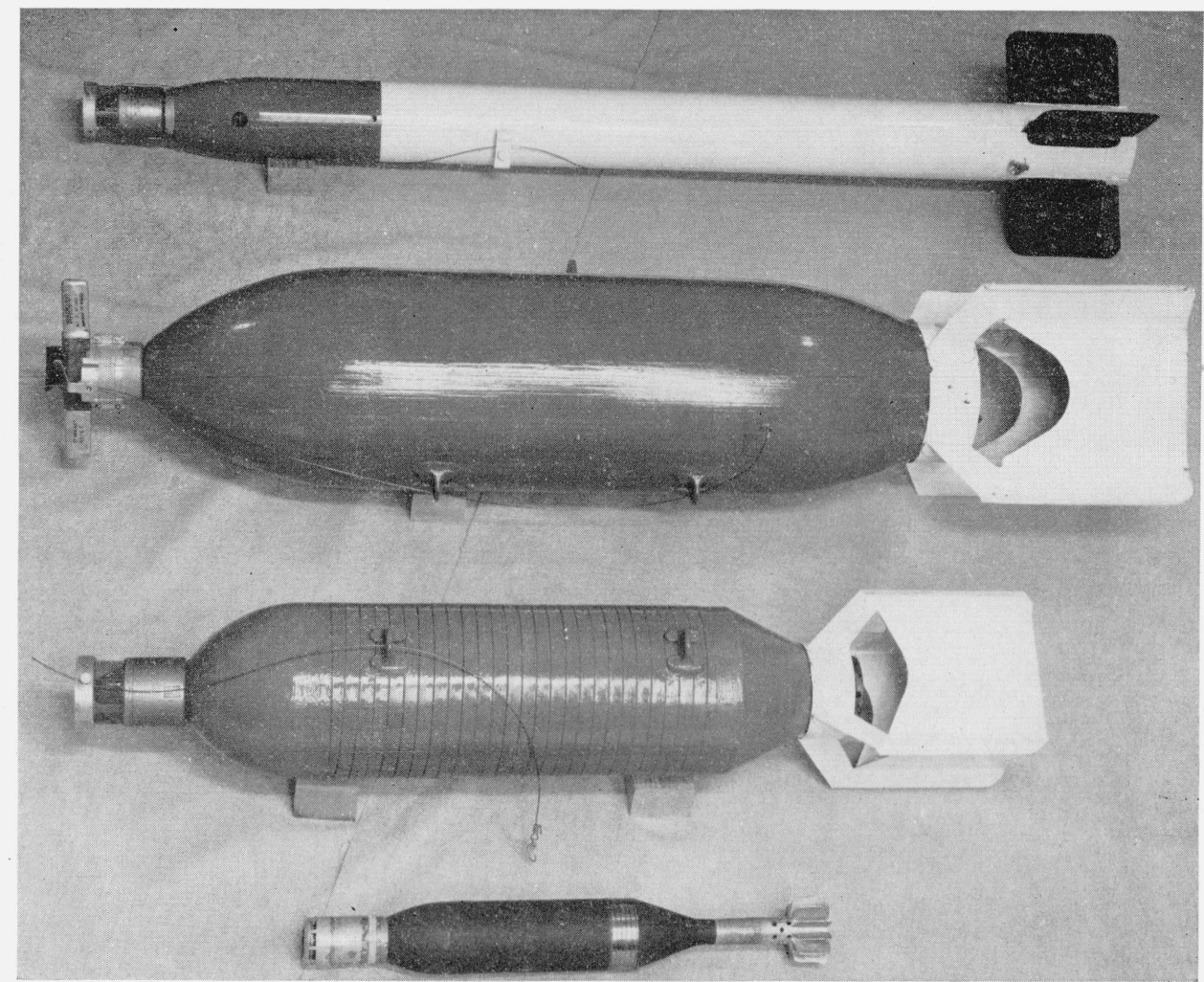

Figure 1.-Radio proximity fuzes mounted on high-velocity aircraft rocket, 500-and 265-lb bombs, and 81-mm trench mortar.

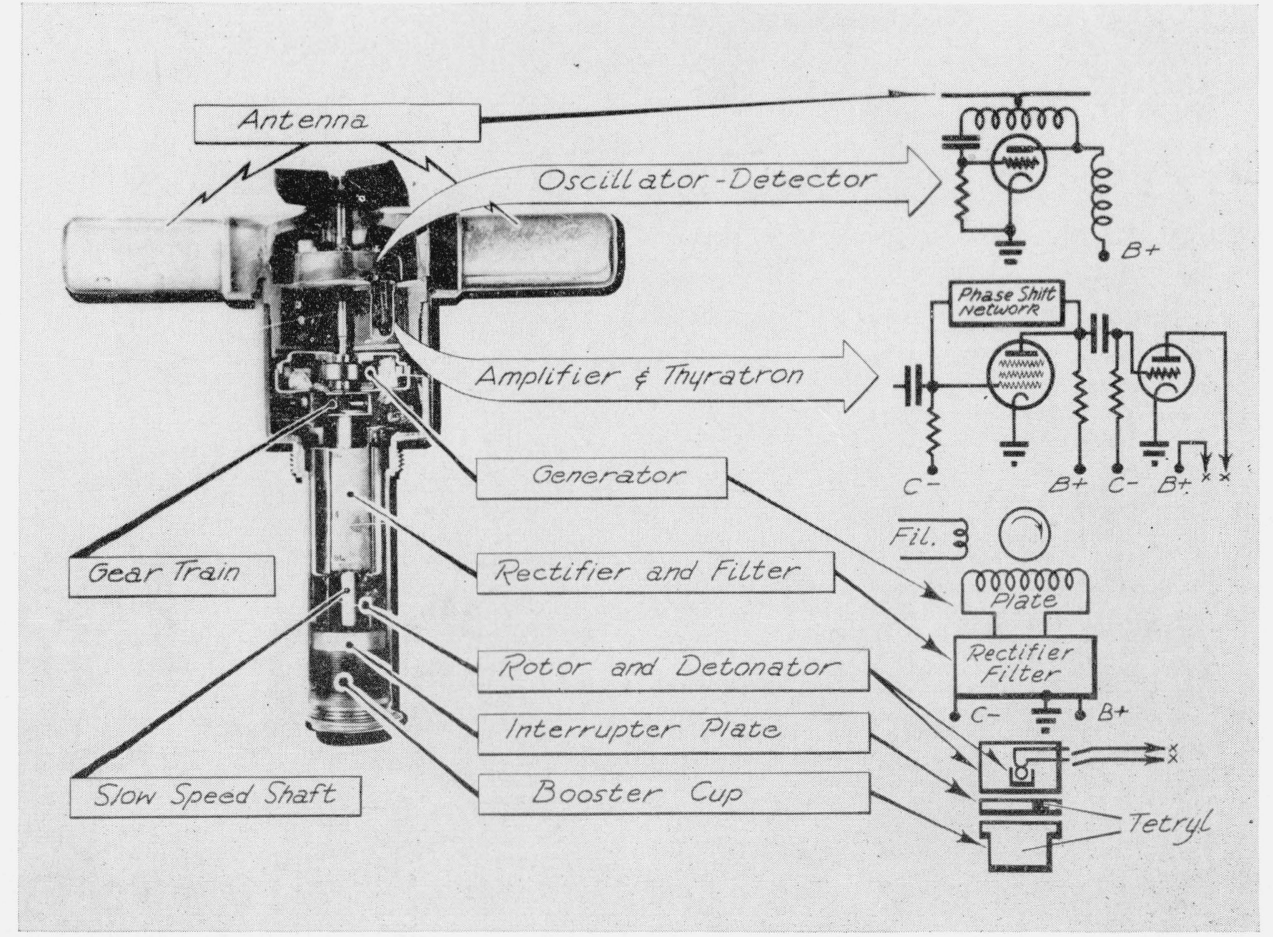

FIGURE 2.-Cutaway of fuze and generalized fuze circuit. 


\section{General Description}

The radio fuze is essentially an oscillating detector. The operating signal is furnished by the combination of the wave reflected from the target with the voltage of the local oscillator. The time of travel of the radiated wave from the oscillator to the reflecting surface, or target, and back results in a phase difference between the transmitted and reflected wave. If the distance to and from the reflecting surface changes, the relative phase changes. When the oscillating detector is approaching the reflecting surface, the phase change appears as an increase in the frequency of the reflected wave, each returning wave front reaching the detector sooner than it would if the oscillating detector were stationary. Thus, the apparent distance between wave fronts is shorter and the frequency is higher.

The actual frequency of the returning wave (since it must go to, and return from, the target) is the outgoing frequency, $f_{1}$, plus 2 (relative velocity/wave length) and the "difference" beat frequency is 2 (relative velocity/wave length). The latter is the signal used to detonate the fuze when it approaches the reflecting target at a preselected velocity (hence beat frequency) and when it is close enough for the reflected wave to have sufficient amplitude.

At useful operating ranges of from 20 to 70 feet, the amplitude of the reflected wave seen by the detector is of the order of a small fraction of a volt. Amplification is necessary to make this voltage large enough to operate the detonator control circuit, usually a thyratron. A single-tube amplifier whose pass band is designed to favor the reflected signal over spurious noise or other signals, is used to increase the amplitude of the detected beat frequency to several volts, enough to cause the standard control circuits to function.

A power supply and a mechanical safety arrangement to prevent premature operation of the detonator completes the primary components of the fuze.

VT fuzes are designed to certain over-all sensitivities which, obviously, involve both oscillator and amplifier design. Greater amplifier gain may completely compensate for a weak or inefficient oscillator detector, but if a weak oscillator is used without a commensurate reduction in the noise voltage (the peak rectified noise voltage at the detector output), the signal to noise ratio is reduced.
Throughout the development and production of VT fuzes, electrical stability in the presence of severe vibration was a prime problem. Although generally called an electronic fuze, the structural and mechanical design required a great deal of attention. Improper design might cause the fuze to operate spontaneously prior to reaching the target. A large part of the engineering and development work was directed toward obtaining the best performance possible, for you cannot drop the same bomb twice. The work was directed along three general lines:

1. Improvement of circuit stability.

2. Reduction of tube microphonics.

3. Reduction of vibration introduced by unbalance in the high-speed generator.

Certain cardinal precepts were established early in the program. Shock mounting was not used because shock mounts have a low natural period with a multitude of harmonics. It was found preferable to make the parts "so rigid that there could be no relative motion." Of course, this really means that the natural resonances of all parts were placed out of the range of the signal frequencies at which the amplifier was responsive. It was accomplished by extremely solid and rugged mounting of all parts and by careful design of all components. Ideally, all parts should be made as a block so that the completed fuze would be literally as solid as a brick. This ideal condition was approached by cementing the oscillator components into cavities in a solid insulator in the form of a disk one-half inch thick, the assembly being tightly fastened to a suitable heavy metal casting. The amplifier was built on a light Bakelite or fiber chassis and set into a cavity in the heavy casting. The cavity was then filled with a potting material so that all parts were fixed in position.

The mounting of the fuze to the projectile was particularly important. For a given vibratory force, acceleration is inversely proportional to mass, and the effective mass is greatly increased if the fuze is made a rigid part of the projectile. Considering the vibration of the rotating system, a firm mounting between the fuze and the projectile did much to reduce its amplitude.

Figure 2 is a cutaway view of one of the bomb fuzes and general circuit diagrams of the primary fuze subassemblies. A more detailed discussion of each of these subassemblies follows. 


\section{Oscillator}

The primary problems of oscillator design are sensitivity, stability, and size. Sensitivity is defined as the absolute change in detected output voltage per unit change in antenna radiation resistance. It may be represented by the equation

$$
\Delta E=\frac{S \Delta R_{A}}{R_{A}}
$$

where $\Delta V$ is the change in detected output voltage, $S$ is the sensitivity, $\Delta R_{A}$ is the effective change in radiation resistance, and $R_{A}$ is the radiation resistance, the antenna circuit being tuned to resonance. In the limit, the equation for sensitivity becomes

$$
S=\frac{d E}{d R_{A} / R_{A}}=\frac{d E}{d\left(\log R_{A}\right)}
$$

The latter form of equation suggests a simple method of measuring the $\mathrm{r}-\mathrm{f}$ sensitivity. Measurements are made of detector voltage, $E$, for various values of radiation resistance, $R_{A}$. The range of radiation resistance is selected to cover the values of all projectiles on which the fuze is to be used.

The next step is to plot the detector voltage against the log of the radiation resistance. The slope of this curve is $d E / d\left(\log R_{A}\right)$ and, therefore, is the $r-f$ sensitivity. Note that the unit of $r-f$ sensitivity is the volt and, again, is interpreted as the number of volts change in detector voltage per unit of change of radiation resistance.
Any oscillating detector will have appreciable $r-f$ sensitivity for a reasonable range of radiation resistances. In a well-designed system, the oscillator is stable and the sensitivity is high for radiation resistance values varying over a large range. This is an important factor in fuze design, since it allows use of the same fuze with a variety of projectiles, small and large. Class $C$ oscillators are generally used because this type gives high sensitivity with good stability and is largely independent of tube characteristics and radiation resistance.

In addition to considering the change in voltage in the presence of a change in radiation resistance, one must consider the absolute value of detector voltage, $V$. For a given sensitivity, the lower we make the value of $V$, the lower will be the noise voltage. Any circuit which increases the sensitivity to noise ratio is to be preferred, even though the absolute signal voltage is low, for low signals are easily amplified.

Note, then, that the radio fuze does not require a detector which is sensitive in the usual sense; $i$. e., responsive to extremely weak signals. Efficiency is a better word; that is, we are interested in the percentage of the voltage developed by the oscillator that appears at the detector output when the oscillator approaches to a fixed distance from a given type of reflector. The higher the percentage, the more stable is the fuze.

\section{Amplifier}

Fuzes have been designed to operate at distances up to 70 feet against aircraft and up to several hundred feet over ground. At these distances, the reflected signal is a small fraction of a volt. This low voltage does not provide a safe margin for controlling the gas relay (thyratron) used to detonate the fuze, and so it must be amplified to a reasonable working voltage. While this is the primary amplifier function, other considerations apply, without which satisfactory operation could not be attained.

The first of these is discrimination against microphonics and other stray noise. The Doeppler frequencies lie in a rather narrow band for any individual fuze and projectile, $(F=2 \mathrm{~V} / \mathrm{\lambda})$. Bomb velocities, for instance, vary from about 400 to 1,000 feet per second, depending on the altitude from which the bomb is dropped, while rocket velocities of projectiles used in World War II varied from 1,000 to 1,800 feet per second. Frequencies other than the corresponding Doeppler frequencies play no useful role in the radio fuze, and every effort is made to reject them through the use of band pass amplifier circuits.

The use of wind-driven alternators (described later) gives an additional reason for restricting the band width of the amplifier. Many fuzes are required to be operative in less than a second so that directly heated filaments must be used. By keeping the alternator frequency outside of the Doeppler range, the frequency cutoff of the amplifier discriminates against the hum in the a-c filament supply to such an extent that filament center tapping is not needed. 
Another consideration is the class of target. There are two general types - the ground and the aerial target. The ground is considered to be a level plane, and the Doeppler frequency is proportional to the vertical component of velocity. The aerial target as represented by the airplane has a complicated reflection characteristic whose reflecting power can be roughly compared with that of a tuned half-wave dipole insofar as general characteristics are concerned. The actual reflecting power is determined by a series of field measurements.

To understand the factors affecting the optimum gain frequency characteristics of amplifiers, it is necessary to consider the directivity, or radiation characteristic of the antenna. Nearly all radio fuze antenna systems are rather highly directional. Since the same antenna is used for transmitting and receiving, the sensitivity characteristic is the square of the radiation characteristic. Projectiles approach the ground at a variety of angles and the fuze should not detonate the projectile at heights above ground which are sharply dependent on the angle of approach; a uniform burst height is desired.

Depending on the type of antenna, the gainfrequency characteristic is adjusted to give a uniform height of function for the fuze for any angle and velocity of approach to the target. The two most common antenna types are the longitudinal, employing the body of the projectile as the antenna, ${ }^{1}$ and the transverse, employing a bar as a dipole disposed at right angles to the projectile axis (fig. 1). Longitudinal and transverse directivity envelopes, i. e., for ring-type and bar-type fuzes, respectively, mounted on bombs, are shown in figure 3. Minimum radiation occurs, of course, on the axis of the antenna
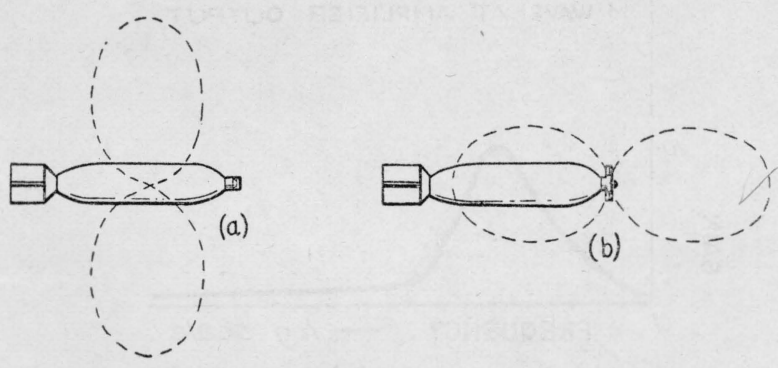

Figure 3.-Radiation patterns for bomb fuzes.

(a) Ring-type, (b) bar-type fuze.

${ }_{1} \mathrm{r}-\mathrm{f}$ energy is end-fed to the body through the antenna ring or eap of the uze. in each case and maximum radiation is roughly at right angles to that axis.

For the longitudinal type, it is obvious that the radiation is least for a vertical drop, and it approaches a maximum as the angle with respect to the vertical is increased. Now, as the vertical component of velocity is highest for high-altitude bomb releases, the angle with the vertical is small, the radiation is low, and the Doeppler frequency is high. For this condition, high amplifier gain compensates for the low power radiated toward the target. Conversely, for low-altitude bomb releases, the vertical velocity is low, the angle with the vertical large, and the Doeppler frequency low. Here, less amplifier gain compensates for the higher power radiated toward the target. By proper design of the amplification characteristic, the height at which the fuzed projectile functions may be held quite uniform, regardless of the altitude of release. This will be treated in more detail later.

The second type, the transverse antenna, has a substantially constant radiation toward the ground for all except extremely low altitude releases, where the angle of fall with respect to the vertical is very large. The amplifier for this type fuze has essentially constant gain over the corresponding range of Doeppler frequencies. Figure 4 shows representative gain-frequency characteristics for the two types of fuzes.

(a)

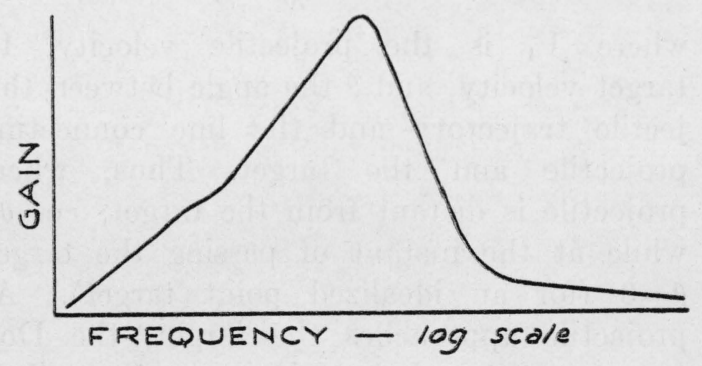

(b)

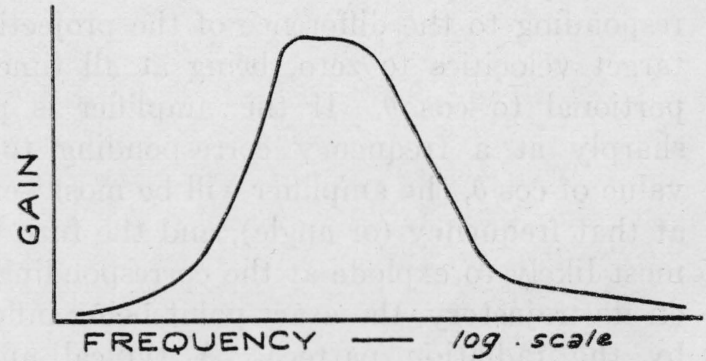

FigURe 4.-Typical amplifier characteristics.

(a) Ring-type fuze, (b) bar-type fuze. 
The radio fuze for aerial targets presents problems in amplifier design that differ from those of the application over ground. The requirement is that the fuze must function at the optimum point as it passes the target. The fragments of an exploding projectile have two velocities - one due to the projectile motion and the other due to the explosion. The greatest concentration of fragments is at right angles to the path of travel of the projectile axis (statically). The projectile velocity combines with the velocity generated by the explosion to cause the equatorial spray of fragments to move forward of the normal to the projectile. Thus, the optimum point for detonation is that where the line from the projectile to the target makes an angle of from 15 to 30 degrees with the normal to the trajectory.

The preferred type of antenna for aerial targets is that using longitudinal excitation, because radiation reaches the target only when the projectile commences to pass it. Consideration of the Doeppler signal pattern, commonly called the $M$ wave, as a fuze passes an aerial target will show the means of using the amplifier characteristic to control the burst point (fig. 5, a). Consider a projectile approaching an aerial target from a distance. The Doeppler frequency depends on the relative velocity of the projectile and the reflecting target. For a target and a projectile traveling in the same direction,

$$
F=2 \frac{\left(V_{1}-V_{2}\right)}{\lambda} \cos \theta
$$

where $V_{1}$ is the projectile velocity, $V_{2}$ the target velocity, and $\theta$ the angle between the projectile trajectory and the line connecting the projectile and the target. Thus, when the projectile is distant from the target, $\cos \theta \cong 1$, while at the instant of passing the target, cos $\theta=0$ (for an idealized point target). As the projectile approaches the target, the Doeppler frequency changes continuously from that corresponding to the difference of the projectile and target velocities to zero, being at all times proportional to $\cos \theta$. If the amplifier is peaked sharply at a frequency corresponding to some value of $\cos \theta$, the amplifier will be most sensitive at that frequency (or angle), and the fuze will be most likely to explode at the corresponding point on its trajectory, the exact point being influenced by the radiation pattern. A typical amplifier gain-frequency characteristic is shown in figure 5 , c.
The $M$ wave signal at the amplifier output is shown in figure $5, \mathrm{~b}$. This is the $M$ wave, of figure 5 , a, modified according to the amplifier gain for the varying frequency.

Most of the amplifiers use only one tube. The high degree of selectivity is shown in figure 4 . It is obtained through the use of strongly regenerative-degenerative circuits. Noise or hum voltages large enough to overload the amplifier may appear at the input, but by feeding the corresponding out-of-phase voltage back from the output, the undesired voltages are bucked out and suppressed.

Conventional resonant L-C circuits are used in combination with the feedback circuits to provide the flat-top band pass characteristic needed for the transverse-excitation type of fuze. The practice of tuning the resonant circuit to the lower limit of the desired frequency band was widely used. Other types of circuits are, of course, possible, but space limitations and circuit complexity prejudiced their use.

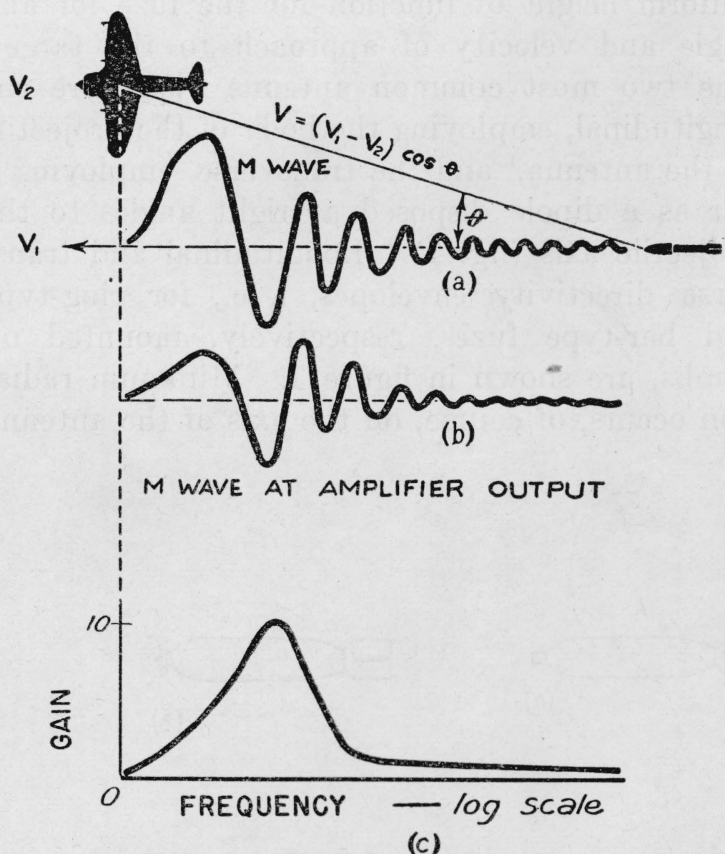

Figure 5.-Reflected signal from aerial target and amplifier characteristic.

(a) Reflected signal, (b) reflected signal altered by fuze amplifier, (c) frequency characteristic or fuze amplifier. 


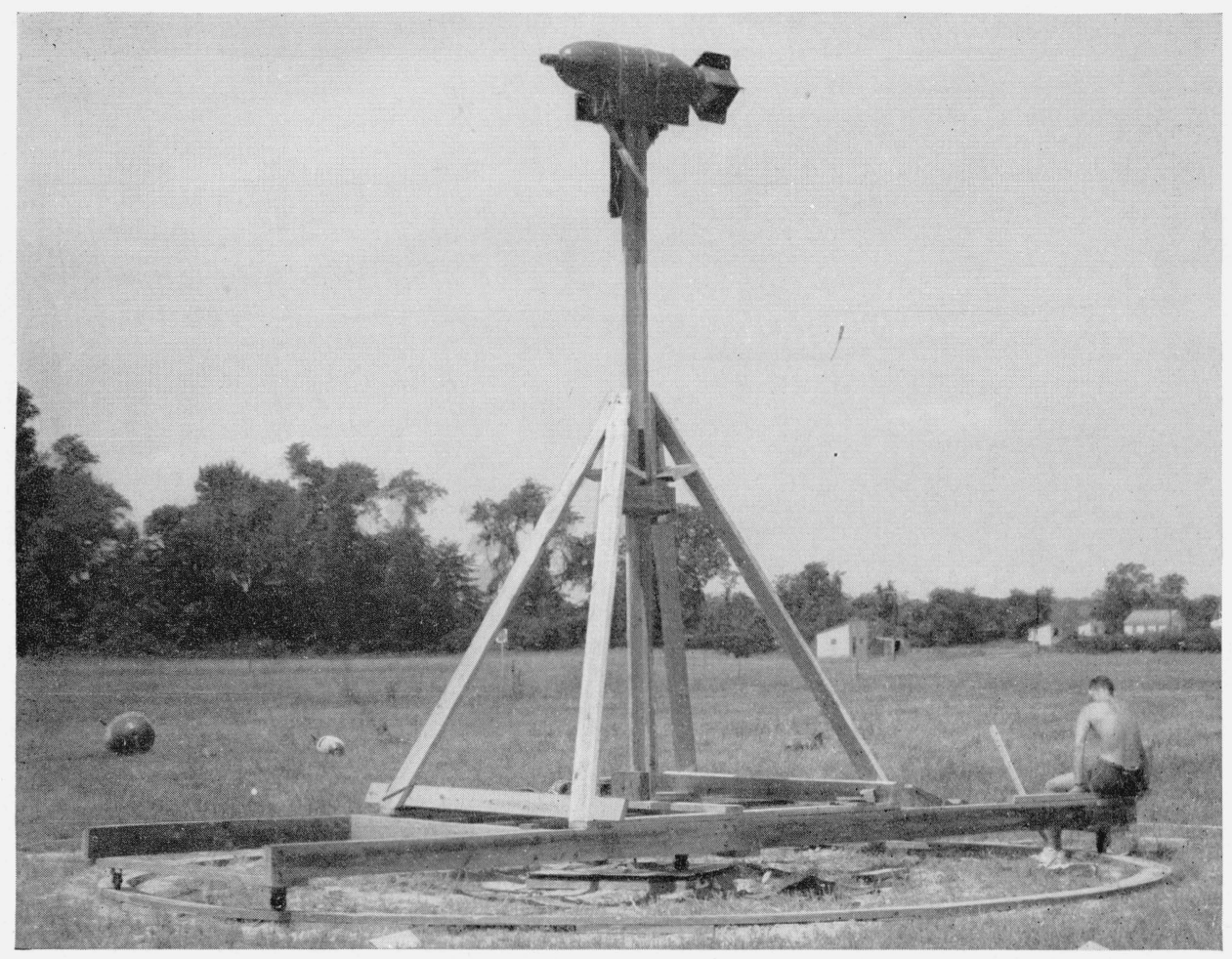

FIGURE 6.-Test structure for obtaining fuze directivity envelope.

POWER SUPPLY ASSEMBLY

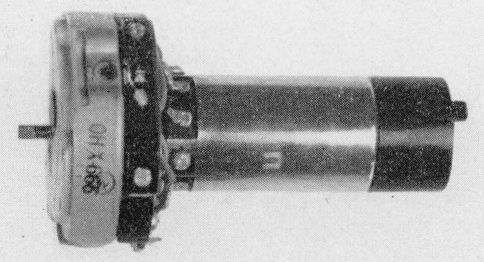

GENERATOR

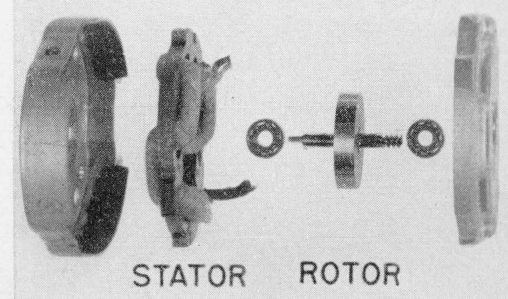

REGULATION NETWORK
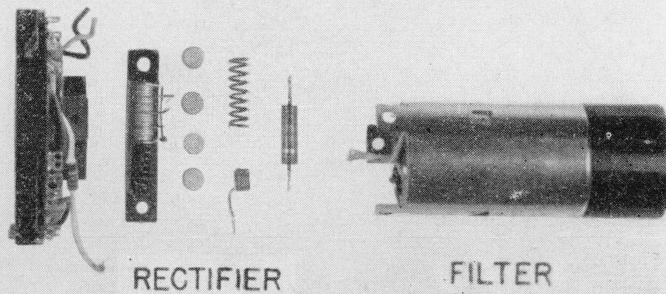

FILTER

Figure 7.--Power supply for bomb fuze. 


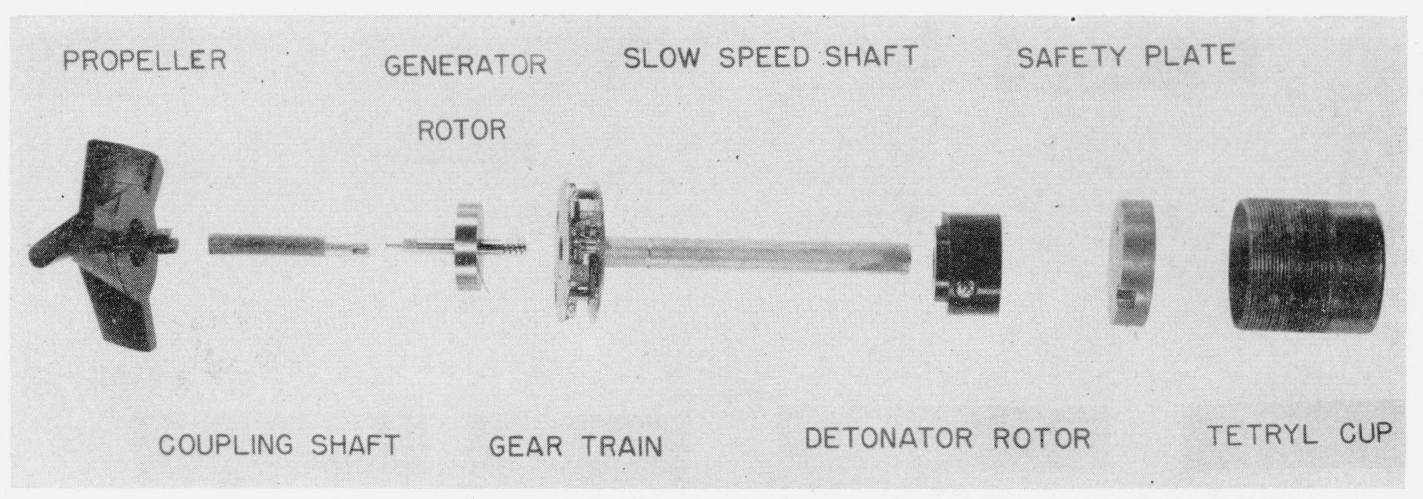

FiguRe 9.-Mechanical drive and arming system.

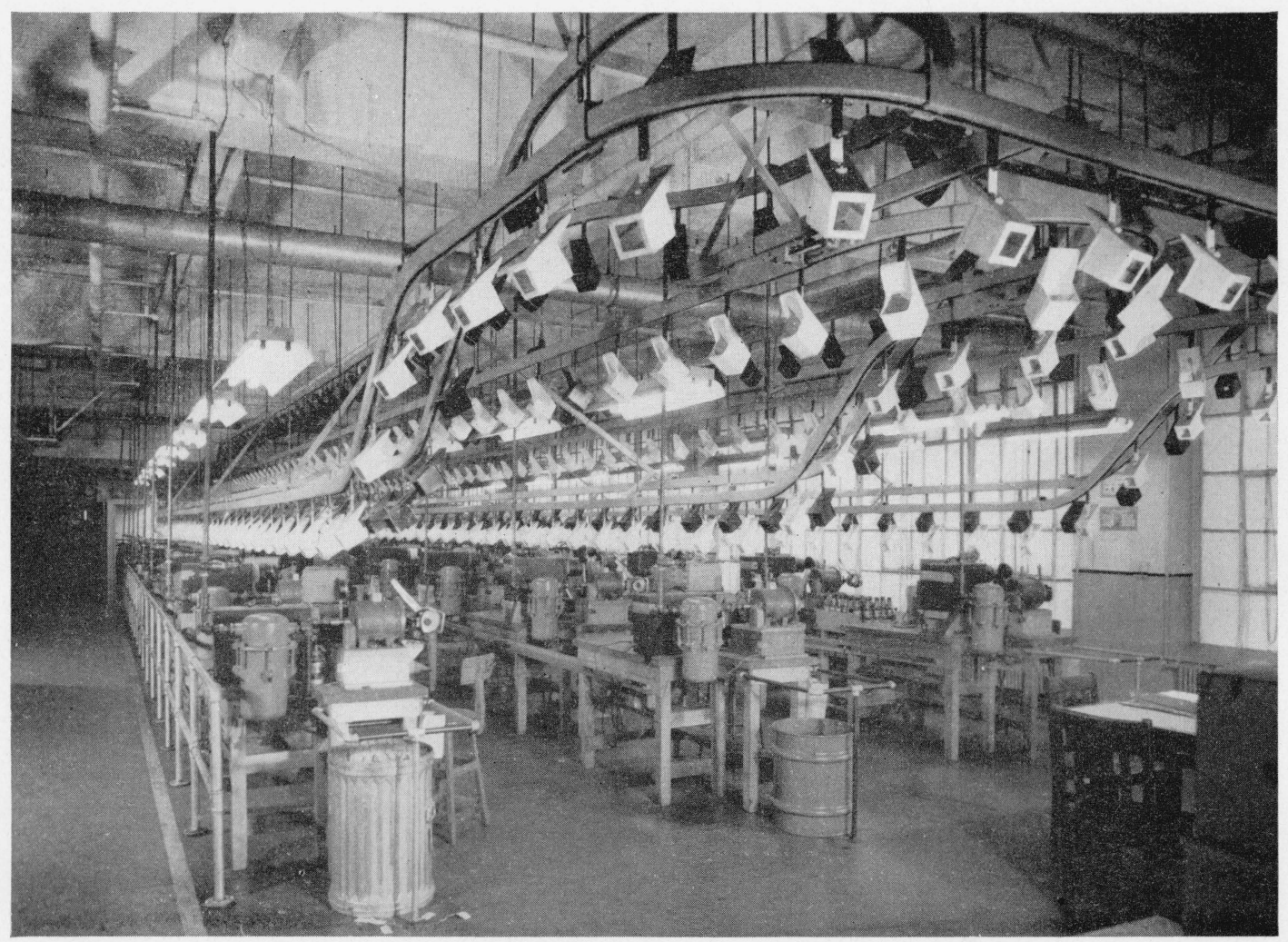

Figure 10.-Fuze production line. 


\section{Radiation, Reflection and Operating Range}

The distance at which a reflected signal is large enough to operate the radio fuze and explode the projectile is dependent on several factors, both internal and external to the fuze. The internal factors that control the height of function are r-f sensitivity, over-all amplification, shaping of the amplifier, thyratron characteristics, and the time delays in all the electrical and explosive paths in the device. External to the fuze, controlling factors include the radiation pattern of the projectile acting as an antenna, external geometry of the fuze-projectile combination, radio frequency, reflection coefficient of the target, geometry of the target, angle of approach, and the relative velocity of projectile and target.

Although the above factors present a most unwieldy number of variables, it has been possible by a combination of theory with laboratory and field experiments to arrive at formulas for computing burst distances with considerable accuracy. The general method of solution is the same for any type of target provided some knowledge of the reflecting properties of the target is at hand. For an aerial target, the reflection coefficient, both in magnitude and phase, as a function of aspect angle, may be determined experimentally. Actually, however, the reflection coefficient of such targets varies so much with angle of approach and type of construction, i. e., metal, plastic, etc., that average figures are assumed for various targets and used for all computations regarding this target. Thus, for example, a certain type of airplane might be equivalent in reflecting properties to five half-wave electric dipoles (i. e., the reflection was five times that of a tuned half-wave doublet oriented for maximum reflection).

In the case of a bomb approaching level ground at an angle, the first step in the calculaion of height of function is to assume the earth to be an infinitely conducting plane. The signal reflected back to the bomb (which we shall call the driving antenna) is the same as that radiated by a mirror image of the bomb (or antenna) located as far below the conducting plane as the real bomb is above it. Thus, the field produced at the driving antenna by the image antenna is calculated in terms of current in the driving antenna, frequency, and geometry. To simplify the remainder of the derivation, the image antenna is now assumed to be a half-wave dipole and the voltage, $e$, induced in it by a given current in the driving antenna is calculated. Applying the reciprocity theorem, the same current at the center of the dipole gives the same induced voltage, $e$, at the feed-point of the driving antenna. Thus, the voltage induced in the driving antenna by the image dipole is found. If this voltage is multiplied by the ratio of the fields produced at the driving antenna by the image antenna and the image dipole, it will yield the true reflected voltage.

The field surrounding the antenna contains three components - the radiation field, the induction field, and the quasi-static field. For many practical purposes, only the radiation field need be considered. This simplifies the calculations and provides an easy working formula for determining operating heights. For the case of a bomb approaching ground at height, $h$, over terrain having a reflection coefficient, $n$, the Doeppler voltage detected by the fuze will be

$$
V_{R}=\frac{k n S}{h \beta_{2}} \phi(\theta) \text { volts (rms), }
$$

where

$S=$ the r-f sensitivity of the fuze,

$k=$ a constant that is principally a function of the wavelength

$$
\beta_{2}=\int_{0}^{\pi} \phi(\theta) \sin \theta d \theta \begin{aligned}
& \text { (this expression may } \\
& \text { be readily computed } \\
& \text { by numerical inte- } \\
& \text { gration) }
\end{aligned}
$$

$\theta=$ the angle of the major axis of the bomb with respect to the vertical.

$(\theta)=$ the directivity envelope (See fig. 3.)

Assume the input to the amplifier required to fire the thyratron is $V_{T}$ volts (r. m. s.), measured at the Doeppler frequency corresponding to the altitude at which the bomb is dropped. ${ }^{2}$ Inserting this value in place of $V_{R}$ in equation (1) yields the height of function.

$$
h=\frac{k n S}{V_{T} \beta_{2}} \phi(\theta) \text { feet. }
$$

All the time delays in the fuze system total less than 5 milliseconds, so that at normal operating

\footnotetext{
$\begin{aligned} 2 \text { Frequency } & =\frac{2 V / \lambda \mathrm{c} / \mathrm{s}}{V}=\sqrt{2 g \text { (altitude) }} .\end{aligned}$
} 
ranges and speeds, they can be neglected without appreciable error.

A structure for obtaining a directivity envelope for a fuze mounted on a 500-pound bomb is shown in figure 6. Provision was made for continuous rotation of the structure. In the figure, the operator is reading the angular position on the ground protractor. The signal radiated by the fuze-bomb combination is picked up by a receiver located approximately 100 feet away. The intensity of the signal is measured for each angular position of the bomb.

\section{Power Supply}

In addition to the requirements of small size and reliable operation at substratosphere temperatures $\left(-40^{\circ}\right.$ C. $)$ and tropical conditions $\left(+60^{\circ} \mathrm{C}\right.$., high humidity), the principal requirements that had to be met in the design of a universal type of power supply for the rocket, bomb, and mortar applications were that it must have a long shelf life, and be a permanent part of the fuze; i. e., should not have to be assembled to the fuze in the field.

A careful study of all methods of power supply led to the selection of a wind-driven a-c generator with a suitable rectifier-filter system as the most practicable solution to the problem. Considerably more than the necessary power to operate such a device was available in the wind stream, and with proper mechanical design, it could be
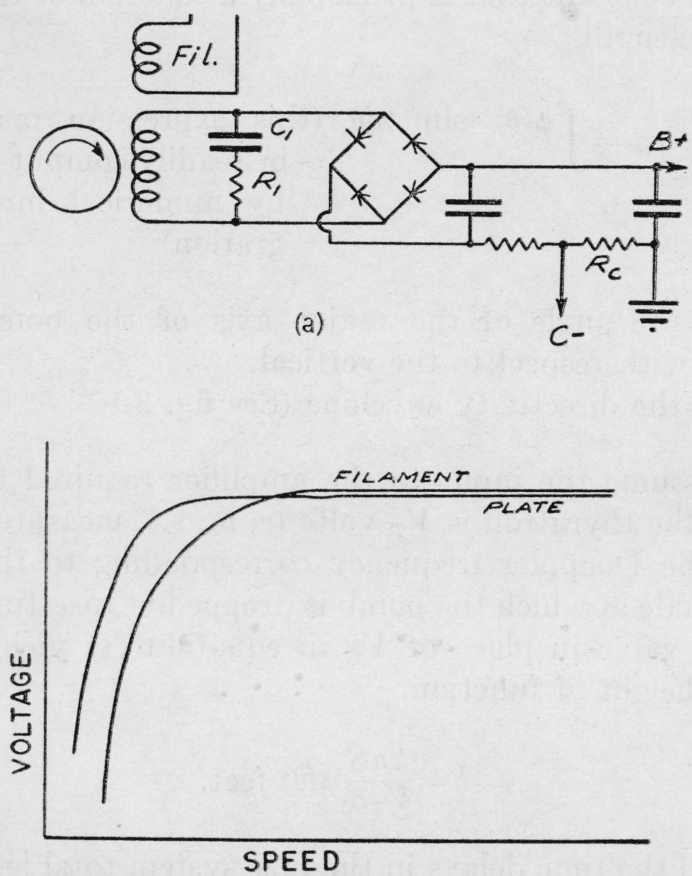

(b)

FIGURE 8.-Generator circuit and characteristic.

(a) Circuit arrangement, (b) voltage-speed characteristic. harnessed without appreciably affecting the ballistics of the projectile. In fact, only a tiny fraction of the power available in the air stream was needed to supply the electrical energy for the fuze. The generator power supply not only met the requirements outlined above, but provided the additional feature of an exceptionally safe method of arming the fuze, to be described later. One of the power supplies used in the bomb and rocket fuzes is shown both in assembled and exploded views in figure 7.

The a-c generator consisted of a six-pole magnet rotating within a stator carrying the coils in which the voltage is generated. The rotor of this alternator was a simple cylinder approximately 1 inch in diameter and one-fourth inch thick. Composed of Alnico, it was magnetized in production by simply inserting it in a six-pole magnetizing fixture and sending a brief pulse of unidirectional current through the six coils on the fixture. For the stator, either a standard six-coil six-pole arrangement or a single-coil sixpole winding, little used in modern practice but of a type described in literature dating back to $1896,^{3}$ was employed. Separate windings were used for the $A$ and $B$ supply. $C$ bias was obtained from the rectified $B$ voltage.

By properly shaping the amplifier gain-frequency characteristic, it was possible to use alternating current directly on the filaments of all tubes. A bridge rectifier was used for the plate and bias supply and filtered by means of a simple R-C filter, as shown in figure 8, a. An extra resistor $\left(R_{c}\right)$ inserted at the low-potential side of the $B$ output provided the necessary $C$ voltage. This allowed the $C$ voltage to be a definite fraction of the $B$ voltage and to vary in the same manner as the $B$ voltage. Thus, some compensation was provided for variations in $B$ voltage from generator to generator in production.

The large leakage reactance of the high-voltage

3 Edwin J. Houston and A. E. Kennelly, Electrodynamic machinery for continuous currents. (The W. J. Johnston Co., 1896.) 
winding was made use of in achieving excellent voltage regulation with speed. In the various projectiles employed, speeds ranged up to 125,000 rpm, the higher speeds occurring in the turbine models. With care in design, the top speeds could be reduced to less than $100,000 \mathrm{rpm}$, but the range was still large. The normal open-circuit voltage of this type of alternator increases almost linearly with speed. The obvious step in obtaining good regulation was to raise the voltage at low speeds by resonating the leakage inductance with an external condenser. This was the method adopted. Using the shunt regulation components $R_{1}$ and $C_{1}$, speed regulation characteristics similar to that of figure 8 , b were obtained. The plateau is reached at a speed below that at which the fuze is armed; that is, at which the fuze is ready to function electrically. The purpose of resistor, $R_{1}$, was to broaden the resonance effect of $C_{1}$ and the generator leakage inductance and thus limit the voltage at resonance to give constant voltage at all speeds above resonance.

By virtue of the inductive coupling between the $A$ and $B$ windings, the impedance reflected into the A voltage circuit, with the $B$ regulation components and proper $B$ load in place, was such as to provide an $A$ voltage speed-regulation curve similar to that of the $B$ circuit.

Full-wave rectification was obtained by means - of selenium rectifiers in the four arms of the bridge. In some applications, a voltage-doubler rectifier circuit was employed, allowing good performance with half the number of rectifier cells.

One of the important factors which helped to make possible the tiny generator-powered radio proximity fuze was the development of small selenium rectifiers. The rectifier itself, in 1 of the applications, was composed of 10 cells, each about one-fourth inch in diamater.

Choice of the selenium rectifier was based on numerous considerations, including both the economical and the physical. Vacuum tubes would have provided a suitable solution to the problem of rectification; however, their cost and difficulties of production, including time, tools, and strategic materials needed, made it imperative than an inexpensive and easily produced substitute be found. Then, too, vacuum tubes draw filament power and in addition had to be made rugged. For example, in the trench mortar application, the fuze is designed to withstand a set-back force of 10,000 times that of gravity.
This set-back occurs as the shell is fired. Although the ruggedness necessary was accomplished in the design of the tubes, in order to obtain it, special features of construction were necessary, with the attendant increase in cost and difficulty of production. It was felt that wherever possible, substitutes for vacuum tubes would be desirable in a device of this type.

Both selenium and copper oxide rectifiers were tested. The latter have especially desirable features and in the early stages of development appeared to be favorably adapted to the problem. In particular, they show a low reverse dynamic characteristic - that is, low conduction of current in the reverse direction. However, concentrated development on the selenium disk soon resulted in the obtaining of reverse characteristics which exceeded those of the copper-oxide rectifier. Also, the copper-oxide rectifier did not perform as well under the extreme ranges of temperature met in practice, especially at low temperatures, and full attention was soon focused on the selenium rectifier.

The exact method of manufacture of selenium cells is regarded as a trade secret. However, a general idea of the method may be told.

The cells are made on a base metal, such as steel, aluminum, etc. A large strip of metal is used and the individual cells are partially punched out, being held to the strip by 0.005 inch of metal, which keeps the cells on that particular strip together throughout all the subsequent manufacturing procedure.

Selenium powder is applied to the strips, which are then baked under pressure at approximately $115^{\circ} \mathrm{C}$. The strips are again put into an oven, this time without pressure, and held at a temperature of slightly over $200^{\circ} \mathrm{C}$. Care is exercised as this is very near the melting point of selenium. A shiny black appearance resulting from the first baking now changes to a crystalline grey. This practice is called annealing.

Following this, the cells are placed over a vapor of selenium dioxide. The material adhering to the selenium forms a thin layer and acts as a catalyst in the formation of the barrier layer. Finally, the counter-electrode is sprayed on, a mask being used to prevent a short circuit at the cell edges. The cells are now electroformed by connecting them to a source of d-c voltage.

The assembly of rectifier cells in their containers. shown in figure 7 , met rigid microphonics tests. 
Impellers of both propeller and turbine type were employed, the former on rocket and bomb fuzes and the latter on the mortar and also on one of the rocket fuzes. The propeller type was either molded of Bakelite or stamped out of steel. The turbine type was die cast. The pitch was selected at a value that gave the best compromise between fast starting and low top operating speeds.

The extremely high rotational speeds at which the generators were operated introduced severe mechanical problems. Even with the small degree of unbalance in early models, strong vibration resulted, and bearings were destroyed in a relatively short time. The introduction of commercial ball bearings in place of the sleeve bearings originally used gave a material improvement in performance.

The rotor, in addition to possessing excellent magnetic properties, required special metallurgical design to withstand the high speeds without tearing apart. Vanes were also balanced. End play in the rotating system was kept to minute figures hitherto thought impossible in small devices designed for very high production rates. For the higher generator speeds, more attention was given to balancing of the rotating system, and operation was so much improved that it was possible to return to the use of sleeve bearings.

\section{Safety and Arming}

The radio proximity fuze, when armed, is sensitive to any sort of motion or action in its neighborhood that will change the amplitude of reflected waves (at a rate at which the amplifier is responsive). One may cause the fuze to operate by waving a Bakelite rod close to it, or by touching two wires together at a considerable distance from the fuze. Touching a metal pipe or any other ground or metal object may change the reflected signal enough to fire the fuze. For this reason, exceptional care must be taken to see that the fuze circuits are not completed until the fuze is well away from the firing or release point. A fuzed bomb or rocket must be many hundreds of feet away from the plane or the ground firing point before there is any possibility of the fuze functioning. Several safety means are employed. (1) A thick metal plate, usually called an interrupter plate, is interposed between elements of the explosive train so that if the electric detonator is discharged prematurely, the main explosive charge cannot be set off. (2) The electric detonator is disconnected from the power circuit. (3) With generator-powered fuzes, there is no power available to set off the detonator until the projectile is moving through the air at a high rate of speed.

Arming the fuze is accomplished through a gear train, driven by the same vane that drives the generator. Rotating the gear train removes the interrupter plate and connects the electric detonator. This occurs after the vane has made a preset number of revolutions corresponding to a fixed distance of air travel. The usual arming distance varies with type of projectile employed and may be as much as three or four thousand feet. Figure 9 includes a break-down of safety devices which, in the unit shown, are built into the power-supply assembly.

The detonator is set off-center in the detonator rotor, and its terminals are connected to contacts on the face of the rotor. When the fuze is not armed, the detonator is misalined a number of degrees from a small hole in the interrupter plate. The hole is filled with tetryl, a material widely used in explosive train elements. The tetryl cup holds a larger charge of tetryl, which is used to detonate the main explosive charge of the projectile. As the vane turns, the reduction gear train slowly turns the rotor by means of a slowspeed shaft. When the detonator is directly above the tetryl-filled hole in the plate, contact with the detonator leads is made; the rotor is released from the slow-speed shaft and locked in place, completing the arming of the fuze.

In using the bomb fuzes, an arming wire attached to the airplane is threaded through a metal pin on the fuze, which is arranged to block the fuze vane from turning. As the bomb is released; the wire is withdrawn, the metal pin falls out, and the vane is free to turn and arm the fuze.

Rocket and mortar fuzes require other safety means. These fuzes have accelerating forces of from 10 to 10,000 times gravity at the moment of firing. Mechanical locks, which release the vane under the forces of acceleration, prevent arming of the fuze except when it is fired. 


\section{Tubes and Other Components}

Much of the effort expended on radio fuzes was directed toward the development and procurement of small and rugged components. Chief among these were the tiny tubes, as no suitable tubes were available at the start of the war. Work was started with various tube manufacturers on improving the hearing-aid types of tubes, the initial steps being taken by the Navy shell fuze development group of the Johns Hopkins Applied Physics Laboratory. Tube work on the Army phase of the fuze program at the same companies was directed primarily toward developing tubes free from microphonics in the presence of severe vibration.

Many new testing methods and procedures had to be developed, along with rigid manufacturing controls. As a result of this work, a very strong and stable line of inch-long tubes about fivesixteenths inch in major diameter were developed and produced in sufficient quantity to meet the heavy production requirements of the proximity fuzes. None of the production programs were ever delayed by lack of tubes.

For most of the other components, such as condensers, resistors, and coils, there was a continuous effort to reduce size and improve performance at extremes of temperature and humidity. Too much credit cannot be given to manufacturers of these items for the manner in which they engineered and produced the large quantities required.

Another important phase of the project was the development of suitable plastic materials for the nonmetallic parts of the fuzes. These parts included the nose cap, oscillator blocks, coil forms, and numerous housings for other components. Rigid mechanical requirements were imposed on the plastics by virtue of the severe strains to which the fuzes were subjected. In addition, those parts located in the high-frequency circuits had to have especially good electrical properties. These problems were solved by the close cooperation of plastics manufacturers.

\section{Production and Laboratory Testing}

Perfection is a prime requirement of ordnance items. A bombing mission may carry 1 or 2 dozen bombs in a round trip of as much as 2,000 miles. Into the venture goes a bomber costing hundreds of thousands of dollars and a crew too valuable to reckon in money. For each fuze that fails to function, one bomb is lost to the mission and the whole mission may be jeopardized.

In order to assure the best possible fuze operation, a rigid system of production and field testing was instituted before release of the fuzes to the war theaters. Each fuze had to pass a comprehensive final test, and each subassembly was carefully checked prior to final assembly. Proper inspection on the line was of paramount importance. Usual production practices for electronic equipment could not be followed, for a device constructed to ordinary camera model radio set specifications could hardly withstand firing in a rocket or mortar.

Special inspection agencies were set up by the Army Signal Corps or Army Ordnance Department in each area of production to further the rigid inspection system.

The assembly lines were either of the conveyor or the pass-along system. Every fifth or tenth operator was an inspector, and a defect in a subassembly could not go far without detection. After passing minute inspection, both mechanical and electrical, samples of units were selected from each lot produced and shipped to the National Bureau of Standards. There they were tested to exhaustion in the specially built Control Testing Laboratory. They were put through life tests, low- and high-temperature tests, temperature cycling from one extreme to another, humidity tests, salt-spray baths, and a very rude jolt test, after which they were required to perform properly. After the units were packed in special hermetically sealed cans for shipment overseas, they were subjected to a packaging test. ${ }^{4}$ This was somewhat akin to being dropped off a truck and jostled around in every conceivable direction hundreds of times. Again the units were expected to perform properly after the test.

At the Control Testing Laboratory, the sample units were disassembled and each subassembly and the principal components subjected to detailed analysis to determine potential sources of failure. Out of these tests came recommendations for

4 By experts at Picatinny Arsenal. 
improvement in design. A special production engineering section was set up at the National Bureau of Standards to act as liaison between development groups, pilot production lines, control testing, and mass-production plants. Valuable trade secrets were exchanged freely between manufacturers in the common interest.

The production line of one of the modern fuze plants is shown in figure $10 .^{5}$ This plant utilized an overhead conveyor system for passing units from the final assembly line to final test, thence into the packing room. A closeup of the conveyor system and a final test chamber is shown in figure 11. A fuze is within the chamber; the end of its power-supply assembly is seen projecting from the chamber face. A jet of high-pressure air drives the vane.

Throughout production, testing of completed fuzes remained the most pressing single problem, principally because of the difficulty of accurately simulating in a test fixture the actual conditions of a projectile in flight.

The final test chamber had to present both an electrical and a mechanical load to the unit. A dummy antenna was used to simulate the radiating body of the projectile. Mechanically the fuze was screwed into a mount that simulated the mechanical condition when screwed into the nose of a bomb, rocket, or mortar. Thus mechanical resonances in the fuze were allowed to play their full part in generation of electrical noise. Not only was the electrical noise measured on final test, but other parameters of oscillator, amplifier, and power-supply performance were determined with close-go and no-go limits. In this manner, uniform operation in the field was assured.

Women operators were generally used to operate these stations. It is a distinct credit to them that they were by far the majority of the workers who built and tested these complex electromechanical devices.

A subassembly test station is shown in figure 12. This station was used to check the $r-f$ and a-f performance of one type of bomb fuze. The fuze head is shown clamped in the reclining side of the cube-shaped metal box. With the side moved up in place so as to close the box, an r-f load of approximately infinite impedance is presented to the oscillator. Any desired load may then be added by connecting a suitable $r-f$ resistor from the oscillator antenna lead to the side of the box.

\section{Field Testing}

Before any lot of fuzes could be accepted for shipment to the field of action, a sample had to pass an Ordnance proving-ground test. In the case of bomb fuzes, they were tested in bombs dropped over land and water. The beight of function was measured and the lot accepted if the units operated within specified limits. The height test was not the only test. Numerous safety tests with and without high explosives were made to assure proper operation of the arming and safety devices.

Interesting proving-ground tests of a fuze for the Army 41/2-inch rocket were carried out at the Div. 4 N. D. R. C. proving ground at Fort Fisher, N. C., near Cape Fear. In the first tests at that station, an attempt was made to use aerial targets held aloft by small barrage balloons. Testing difficulties were many, for a radio fuze is sensitive to its departure from ground as well as to its approach to ground. Furthermore, early rocket projectors and aiming mechanisms were

$\checkmark$ Courtesy Emerson Radio and Phonograph Corporation. crude and did not permit accurate sighting on a target swaying in a wind. It was then realized that if the projectile could be fired with its trajectory parallel to a plane surface, free space conditions would be simulated, and fixed range conditions could be established. Such an arrangement is shown in figure 13. The rocket-firing tower is shown at the left and a simulated bomber at the right. The latter was made of plain chicken wire supported on four telephone poles. As the rockets were fired approximately horizontally, variations in the terrain between tower and target would produce undesired voltage ripples in the output of the amplifier. To eliminate this imperfection, the ground below was leveled off with a bulldozer. In this manner the fuze, which is insensitive to reflections of constant phase, was unaware of the fact that it was not traveling in free space. In figure 13, a puff may be seen below the end of the left wing where a rocket with a radio proximity fuze fired on passing the target. Smoke puffs were used in place of high explosives 


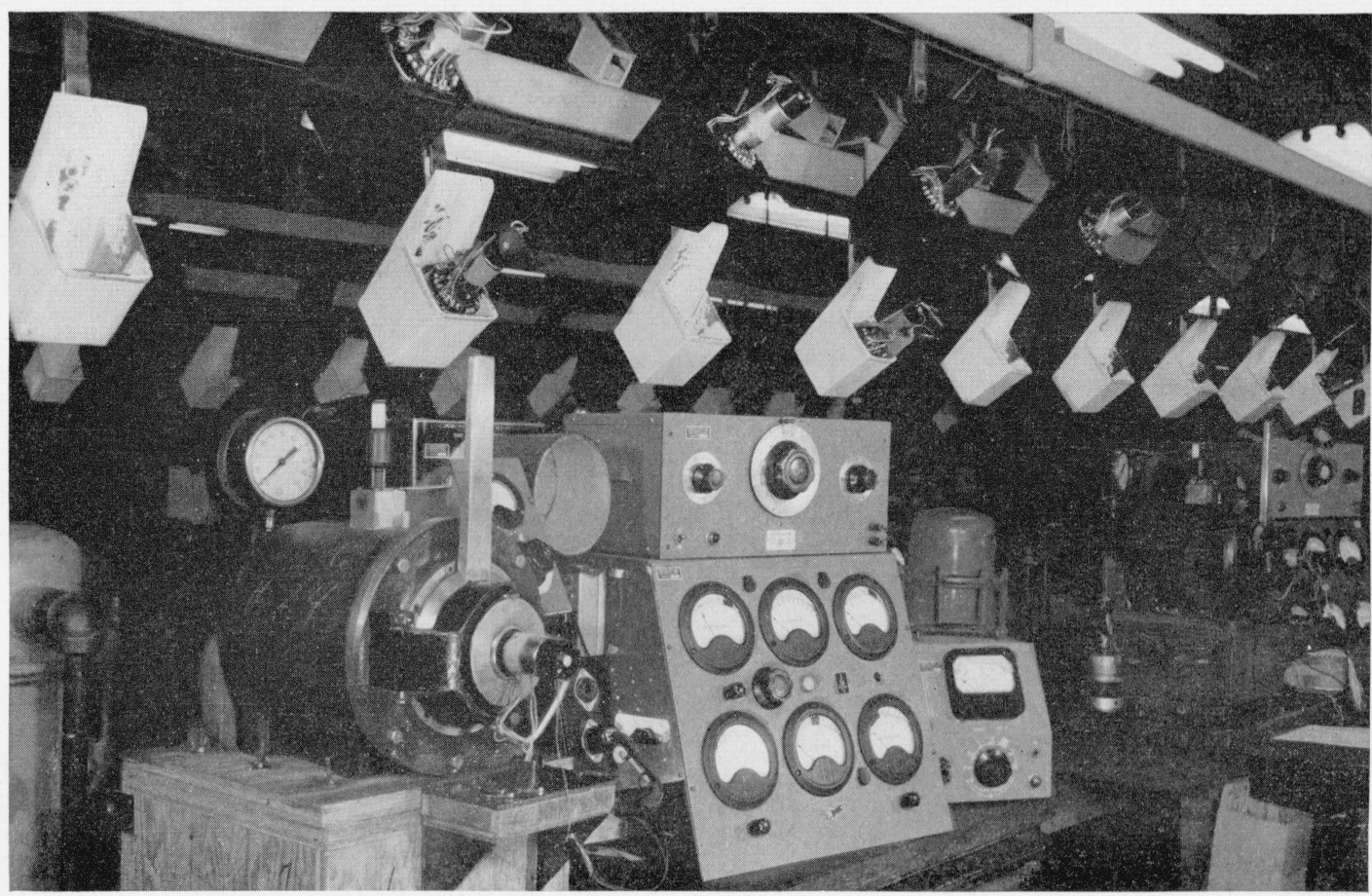

Figure 11.-Final-test station.

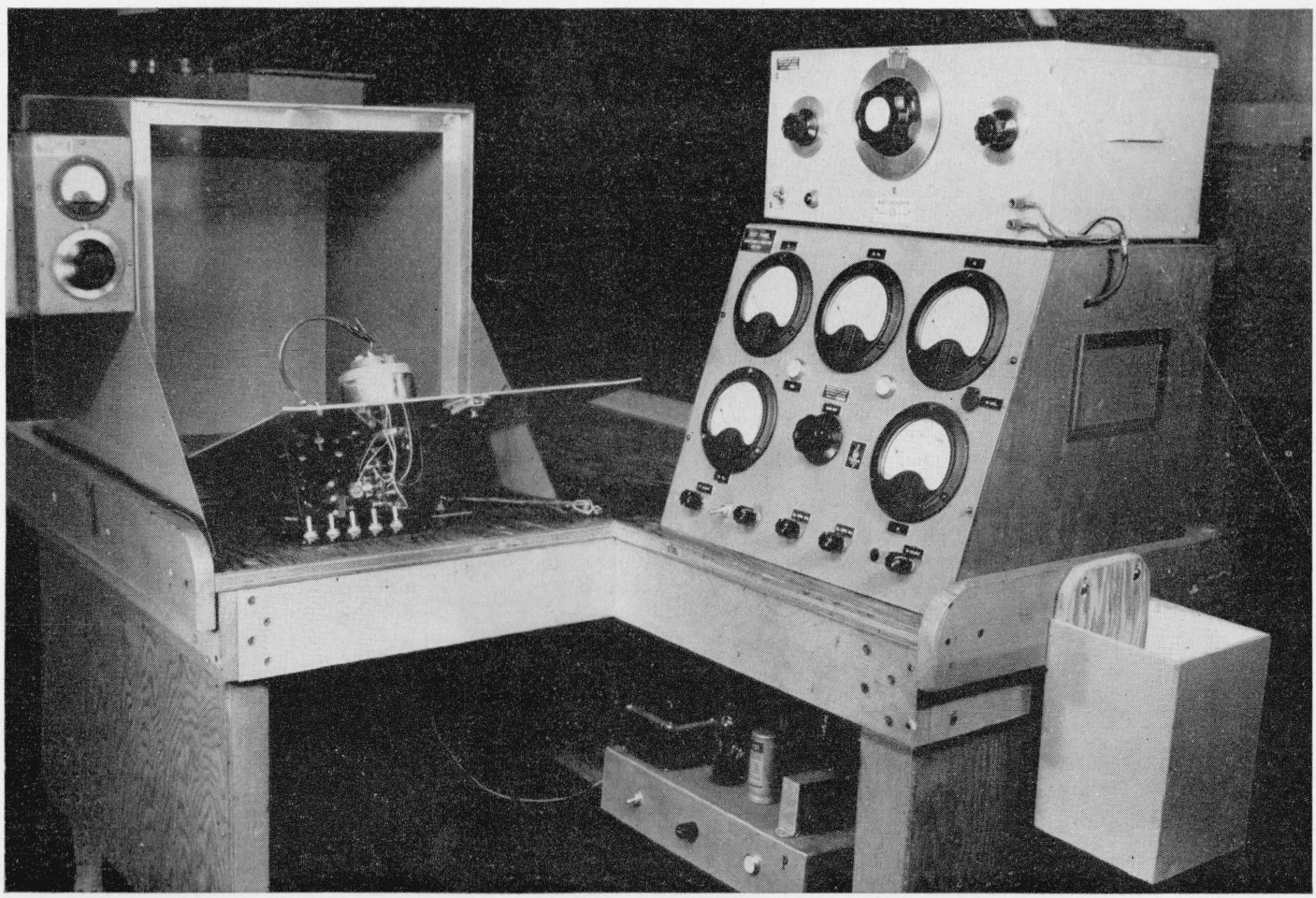

FigURE 12.-Subassembly test station. 


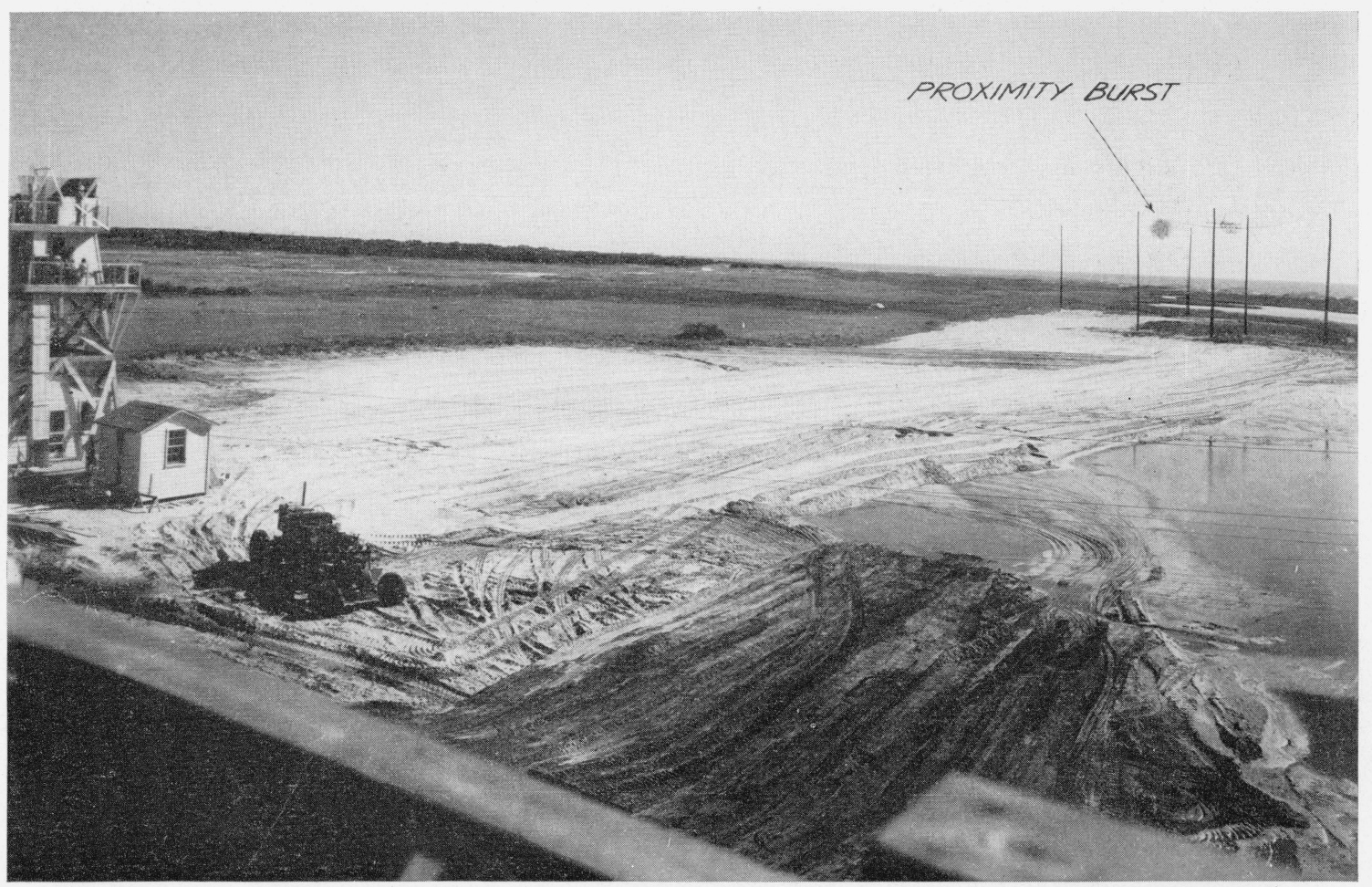

Figure 13.-Target range at Fort Fisher, N. C. 
in the rocket to indicate the point of burst and avoid replacing the target after each shot. On passing the target, the rocket continued on into the waters of the Atlantic Ocean, which may be seen on the horizon.

In a treatment of this type, only a glimpse may be had of the large amount of testing that was carried out on the radio fuzes in proving in the design and quality of manufacture and determining the performance in the field. Thousands of acceptance tests were made at many proving grounds in this country, and innumerable operational tests were made here, in England, and on the continent. Added to this was the valuable information yielded by the enemy, who, so obligingly, supplied targets for numerous tests under actual battle conditions.

The program was conducted under the sponsorship of the Army Ordnance Department and the
National Defense Research Council, and with the assistance of the Signal Corps.

The radio fuze development and production programs were successful because of the high order of cooperation between military and civilian Government agencies and the American manufacturers. The free exchange of information, even between normally competing manufacurers, contributed immeasurably to good fuze design and production. Principal manufacturers of bomb, rocket, and mortar fuzes were Emerson Radio \& Phonograph Corporation, Friez Division of Bendix Corporation, General Electric Co., Globe-Union, Inc., Philco Corporation, Western Electric Co., Westinghouse Electric \& Mfg. Co., The Rudolph Wurlitzer Co., and Zenith Radio Corporation. Principal tube manufacturers were General Electric Co., Raytheon Mfg. Co., and Sylvania Electric Products, Inc.

Washington, February 7, 1946 